\title{
THE NEGATIVE EXPONENTIAL DISTRIBUTION AND AVERAGE EXCESS CLAIM SIZE
}

\author{
G. C. TAYLOR
}

Consider a claim size distribution with complementary d.f. $H($.$) . Let E(x)$ denote the average claim payment under a policy subject to this claim size d.f. but with a deductible of $x$. That is

$$
E(x)=E \max (0, X-x),
$$

where $E$ is the expectation operator and $X$ is the random variable claim size before application of the excess.

It is well-known-see BenkTANDER and SEgerdahl (1960, p. 630)-that:

$$
E(x)=\int_{x}^{\infty} H(y) d y / H(x)
$$

It was shown by them that $E(x)$ is a constant for all $x \geqslant 0$ if and only if the claim size d.f. is negative exponential:

$$
H(x)=e^{-k x}, x>0, k \text { const. }
$$

This property of the negative exponential distribution is closely related to the fact that it is the only distribution with constant failure rate. See KavFMANN (1969, pp. 20-22).

The constancy of average excess claim size with varying deductible can be useful in practice. For example, if the distribution of motor vehicle (property damage) claim sizes can be assumed roughly exponential, which will often be reasonable, then a variation in the deductible will not induce any variation in the average size of claims paid by the insurer, i.e. after application of the deductible. This will be a particularly useful piece of information if for example one is examining trends in average claim size over a period during which a change in deductible occurred.

Although, as suggested above, it may well be reasonable to assume that claim size d.f. is roughly exponential, a closer examination is likely to reveal that this assumption tends to break down to some extent for large claim sizes (say more than twice the mean). In this case it will be useful to know the conditions on the claim size d.f. which lead to constant average excess claim over only a subinterval of the whole range of the d.f. It is shown below that once again a distribution which is negative exponential (but this time with a scale constant which is not arbitrary) distribution is the one required. 
We require

$$
E(x)=E \text {, const., for } x \varepsilon[a, b] \text { say, }
$$

It will be convenient to write

$$
E(x)=E(b) \text {, const., for } x \varepsilon[a, b] .
$$

It follows from (1) and (2) that, for $x \varepsilon[a, b]$

$$
\frac{1}{E(b)}=\frac{1}{E(x)}=-\frac{d}{d x} \log \int_{x}^{\infty} H(y) d y \text {. }
$$

Integration over $x$ between $z$ and $b(z \varepsilon[a, b])$ and slight rearrangement yields:

$$
\frac{b-z}{E(b)}=\log \frac{\int_{z}^{\infty} H(y) d y}{\int_{0}^{\infty} H(y) d y}
$$

But $\int_{z}^{\infty}$ can be written as $\left(\int_{z}^{b}+\int_{b}^{\infty}\right)$. Substitution of this in (3) and a little more rearrangement leads to:

$$
\int_{2}^{b} H(y) d y=[\exp (b-z) / E(b)-1] \int_{b}^{\infty} H(y) d y,
$$

whence, by differentiation on each side,

$$
H(x)=K e^{-x / E(b)},
$$

where $K$ is the constant.,

$$
K=H(b) e^{b / E(b)} .
$$

Equation (4) shows that, for deductibles in the range $a$ to $b$, the average claim size in excess of the deductible is insensitive to variation in the deductible only if the claim size d.f. is negative exponential on the interval $[a, b]$. Unlike the case in which the negative exponential distribution can be assumed for the whole of $(0, \infty)$, the scaling factor in our case is not immaterial. In fact, Equation (4) shows that, on $[a, b]$, the complementary d.f. must be proportional to a section of the negative exponential complementary d.f. with mean $E(b)$

\section{REFERENCES}

Benktander, G. and C. O. Segerdahl (1960), On the Analytical Representation of Claim Distributions with Special Reference to Excess of Loss Reinsurance, XVIth International Congress of Actuaries, 626-648.

Kaufmann, A. (1969), Reliability-A Mathematical Approach, Transworld Publishers Ltd. 